\title{
APPLICATION OF EVENT CHAIN METHODOLOGY IN SCHEDULE RISK ANALYSIS
}

\author{
Goran Avlijaš, Miljan Radunović \\ Singidunum University, Belgrade, Serbia
}

\begin{abstract}
Risk management has become one of the key components of successful project management, and quantitative risk analysis an indispensable ingredient of successful decision process. Although academics proposed numerous methods and techniques that determine the probability and impact of project risks, just a few have earned wider application in practice. This paper aims to bring the practitioners closer to event chain methodology, as one of the recent project risk modeling techniques that can simplify and improve accuracy of existing methods at the same time. Theoretical part emphasizes the significance of managing the risk events and event chains that affect projects every day, while practical demonstration and case study approach give insights in capability of event chain methodology and its value in real life situation.
\end{abstract}

Key words: event chain, Monte Carlo simulation, project risk, quantitative risk analysis.

\section{INTRODUCTION}

Risks and uncertainties are an inevitable part of each project, and many of these events are very hard to identify and analyze (Hulett, 2016). The inability to anticipate all project circumstances leads to inaccurate project plans, overruns in terms of cost and schedule, and failure to meet stakeholder expectations. Development of realistic schedule that considers potential threats and uncertainties remains one of the key success factors of any project, and one of the biggest issues when it comes to the project management.

Following the analysis of 318 industrial projects from different environments, Merrow (2011) shows that up to $65 \%$ are considered a failure. Therefore, a huge scope of study is open for the application of risk prevention, as presented in Kardes, Ozturk, Cavusgil, and Cavusgil (2013). The literature has identified a number of reasons for poor schedule estimates and insufficient implementation of risk management methodologies (Intaver Institute, 2011). Wrong estimations can be result of unintentional action (psychological bias) or intentional action (organizational pressure) by project planners, which further lead to inaccurate forecasts (Virine, \& Trumper, 2015). Other biases that can be attributed to project forecasting are the optimism and planning fallacy (Buehler, Griffin, \& Ross, 1994; Lovallo, \& Kahneman, 2003).

A reason for false estimation can be related to selective perception as confirmation bias (Plous, 1993) and use of heuristics as cognitive bias (McCray, Purvis, \& McCray, 2002). Selective perception is tendency of managers to emphasize the evidence that confirm their hypothesis and ignore the opposite (Evans, Barston, \& Pollard, 1983). The availability heuristic relates to situation where decision makers base their judgments on similar good or bad performed tasks that have been previously completed. Anchoring heuristic relates to a tendency to remain close to the preliminary estimate.

The accuracy of project plans can be significantly improved by updating the original plan (Wysocki, \& McGary, 2003). This requires the analysis of uncertainties throughout the complete lifecycle of the project and incorporating new knowledge into the project plan. Major problem with accuracy of estimates is complex relationship between different risks. Risks may occur at different times during the activity execution, one can cause other events, risks can be correlated, and these relations needs to be identified and visualized. Although there are many scheduling techniques that include project 
performance measurement and other analytical techniques, most of them are complex and inconvenient to use.

This paper has a goal to explore the applicability of event chain methodology (ECM) as a technique that can simplify the process of risk and uncertainties modeling and mitigate the negative effect of cognitive, confirmation and psychological bias. ECM promise to improve forecasting accuracy by creating a flexible framework that includes visualization, performance measurement, execution of migration plans, moment of events, correlations between uncertainties and repeated tasks (Intaver Institute, 2011).

\section{FOUNDATION AND PRINCIPLES OF ECM}

Risk management usually implies the use of the workflow and various tools and visualization techniques. Project Management Institute (PMI) defines this process as risk identification, qualitative and quantitative analysis, risk response planning, response implementation, and risk control (PMI, 2017). Certainly, one of the key steps when it comes to development of reliable project schedule is risk identification. Proper risk analysis is impossible without extensive risk identification process.

Project Management Body of Knowledge (PMBOK Guide) recommends the use of different risk identification techniques such as checklists, brainstorming, interviews, assumption analysis, SWOT, root cause identification, and various diagramming techniques (PMI, 2017). Although templates and check lists represent an effective way of identifying risks, most of the project risks are industry specific, so generalization should be avoided. Universal check lists like the one developed by Scheinin and Hefner (2005) can be a beneficial and serve as a good starting point.

When it comes to risk analysis, PMI suggest the use of several techniques, such as expected monetary value, sensitivity analysis, and Monte Carlo analysis. Expected monetary value requires the use of decision tree in order to identify project alternatives and support decision making. Sensitivity analysis determines which risk events have the most potential impact on project schedule. Monte Carlo simulation uses probabilistic inputs to generate the distribution of potential results, which enables the inclusion of risk and uncertainty in the project scheduling (Schuyler, 2001). Monte Carlo analysis and sensitivity analysis form the basis for the application of the ECM.

ECM represents a technique for schedule network analysis that enables modeling and visualizing of risk events. Project schedule is usually altered by a series of risk events that can be interdependent. The focus of ECM is identification and analysis of risk events and situations in which one event causes another (i.e. event chains). The main idea is to detect the events and chains with the most significant impact on project schedule and address them before they severely impact the project. Application of ECM analysis usually includes the following steps (Virine, 2013):

1. Creation of best-case scenario estimates of activity and project duration. Due to overconfidence project managers tend to develop optimistic schedule, which is usually impossible to prevent.

2. Definition of risk events and chains with detailed probabilities and impacts on project schedule. This includes development of risk-breakdown structure with all necessary attributes.

3. Execution of Monte Carlo simulations in order to obtain statistical distributions of key outcomes, such as project duration and percentiles of finish times for project activities.

4. Execution of sensitivity analysis that identifies risk events and event chains with the most significant effect on key variables. Reality check validates proper definition of probabilities.

5. Repetition of analysis and reassessment of risk probability and impact throughout project, inclusion of actual project data and risk occurrence that enables up-to date forecasts of project duration.

ECM is based on six principles that include single and multiple events identification, definition of the moment of event and excitation, visualization of event chains, Monte 
Carlo analysis, sensitivity analysis and measurement techniques (Intaver Institute, 2011). Most of these are illustrated by Fig. 1, which is followed by an explanation. Although ECM is considered a relatively modern concept in project management, it uses some traditional quantitative methods such as Monte Carlo and sensitivity analysis, Bayesian theorem, as well as some terminology from the quantum mechanics ground and excited states. ECM is already fully or partially implemented in several software application (Virine, \& Trumper, 2015).

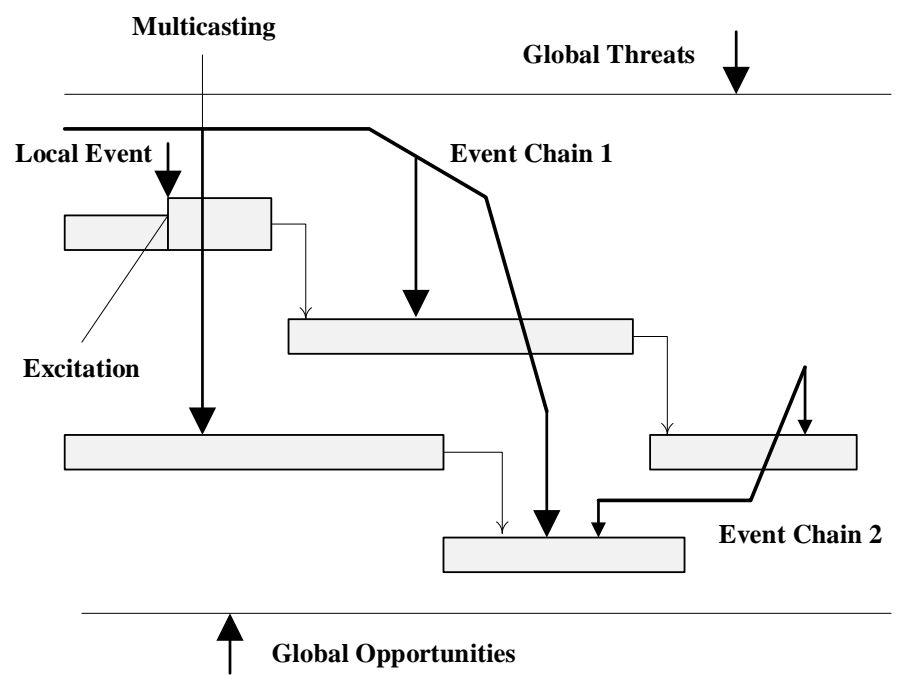

Figure 1: Example of an event chain diagram

1. Moment of event and excitation - most of the real-life project activities are not a uniform and continuous and they are impacted by events that transform them from one state to another. These events include changes in resources, material or work that may alter one or more activity's duration. Assignment of event to activity includes subscription of the impact, probability of occurrence, excited state and the moment of event, which can be also probabilistic. Events can be positive or negative, and it can transform activity from ground to excited state, as well as vice versa (mitigation efforts).

2. Event chains - event chains occur when one risk event (sender) causes another event (receiver), which usually lead to creation of ripple effect through the project and significant impacts. An event can cause multiple events in different project activities or can impact different resources, creating a multicasting effect. Each receiver can act as sender as well, if it triggers another event.
3. Event chain diagrams - event chain diagrams enable visualization of complex relationships between events and schedule, and they are used for the significant events only (Virine, \& McVean, 2004; Virine, \& Rapley, 2003). Event chain diagrams builds on original Gantt charts with specified modifications (Fig. 1). Some of the basic rules that can be easily comprehended include: down arrows represent negative impacts, up arrows positive impacts, individual arrows represent events, multiple connecting lines represent multicasting, arrows outside the chart represent global events affecting all activities, arrow size represents the relative probability of an event, horizontal position on the bar represents the mean moment, etc.

4. Monte Carlo analysis - Monte Carlo simulation is performed to quantify the cumulative impact of the identified events and event chains (Avlijaš, 2019). Beside probability distributions and impacts of risk events, inputs also include the probability distributions and impacts that relate to fluctuations 
in activities duration, which should not have the same root cause as the events to avoid double count of risk. In addition to traditional Monte Carlo analysis results, ECM includes calculation of success rates, probabilistic cash flows, conditional branching, etc.

5. Critical event chains - risk events and event chains with the most significant impact are called critical. Sensitivity analysis is used to identify critical events and mitigate their negative impacts by analyzing the correlations between event chains, project duration and cost. Critical events can be visualized by using a sensitivity chart, that enables calculation of correlation coefficients, event costs and total project cost with risks and uncertainties.

6. Performance measurement - Using of actual performance data ensures the use of updated information and recalculation of probabilities of occurrence and moments of events. This further enables creation of updated project schedule and durations, which is usually accompanied by charts that illustrates variation of chances of completing a project within specific deadline. The chance to meet the deadline is constantly updated and it usually decreases over time due to different project risks, but it can also improve due to risk mitigation actions performed by managers.

\section{ILLUSTRATIVE EXAMPLE}

A simple case will be used to illustrate how risk events can be managed with the use of event chain methodology. Illustrative project consists of three activities (A, B, C) with finish-to-start (FS) relationships. In order to examine different implications, this sequence is presented in four different scenarios: base case, single risk event, multiple independent risk events, and event chain. The data given in Table 1 provides information related to project activities and their attributes in terms of duration and dependencies. Table 2 provides information on probabilities and impacts of risk events that extend activity durations. Generally, events can not only affect schedule, but also cost, safety, performance, quality, technology, and other objectives (Agarwal, \& Virine, 2017).

Table 1: Activities, dependencies and durations for the sample project

\begin{tabular}{|c|c|c|c|c|c|}
\hline \multicolumn{3}{|c|}{ Activity attributes } & \multicolumn{3}{|c|}{ Duration (Base case scenario) } \\
\hline Activity & Predecessor & $\begin{array}{c}\text { Expected } \\
(\mathrm{Te})\end{array}$ & $\begin{array}{l}\text { Optimistic } \\
\text { (a) }\end{array}$ & $\begin{array}{c}\text { Most likely } \\
\text { (m) }\end{array}$ & $\begin{array}{c}\text { Pessimistic } \\
\text { (b) }\end{array}$ \\
\hline A & - & 5 days & 4 days & 5 days & 6 days \\
\hline B & A & 4 days & 3 days & 4 days & 5 days \\
\hline $\mathrm{C}$ & B & 3 days & 2 days & 3 days & 4 days \\
\hline
\end{tabular}

Table 1 provides three-time estimates (optimistic, most likely and pessimistic) which indicate the risk level for each activity. These are used to calculate expected activity durations (Te), and the formula is based on the beta statistical distribution $(\mathrm{TE}=(\mathrm{a}+4 \mathrm{~m}+$ b)/6). Beta distribution is generally used more often than a normal distribution, as it is very flexible in form and can deal with extremes (e.g. when $\mathrm{a}=\mathrm{m}$, or $\mathrm{b}=\mathrm{m}$ ). Before running the Monte Carlo simulation, assumptions for the activities and risks need to be defined.
Definition of assumption includes selection of suitable probability distribution and definition of parameters. This was done separately for the regular variation in activity duration and risk events. For the duration of project activities BetaPERT probability distribution was used. BetaPERT represents a special case of the beta distribution, which unlike triangular distribution uses three parameters ( $a, m$, and $b$ ) to create a smooth curve that fits well to the normal or lognormal distributions (Davis, 2008). 
Table 2: Risks and scenarios for the sample project

\begin{tabular}{|c|c|c|c|c|}
\hline \multicolumn{2}{|c|}{$\begin{array}{c}\text { Activities and risk } \\
\text { events }\end{array}$} & $\begin{array}{c}\text { Single event } \\
\text { prob. for scenario } \\
\mathbf{2}\end{array}$ & $\begin{array}{c}\text { Multiple events } \\
\text { prob. for scenario } \\
\mathbf{3}\end{array}$ & prob. for scenario 4 \\
\hline Activity & Impact & $30 \%$ & $30 \%$ & $30 \%$ \\
\hline A & 1 day & $0 \%$ & $30 \%$ & $30 \%$ (100\% in case of risk A) \\
\hline B & 2 days & $0 \%$ & $30 \%$ & $30 \%$ (100\% in case of risk B) \\
\hline C & 3 days & $0 \%$ &
\end{tabular}

For the risk events custom distribution was used. This type of distribution represents a unique situation and relies on single values, discrete ranges, or continuous ranges. In this case, each risk events were assigned a discrete probabilities and possible impacts of 1,2,3 days, respectively for each activity. Oracle Crystal Ball software was used for the simulation. For each input software selects samples from the assumed distributions, inserts these into mathematical model for thousands of times and calculate distribution of the outcome variable (Williams, 2004).

As can be seen from Table 1, the critical path is simple, and it consist of activities A-B-C. The shortest period in which illustrative project with expected activities duration (Te) and predefined dependencies can be completed is 12 days. For each of 4 scenarios 1,000 trials were simulated, and the resulting statistical distribution of project duration is provided in form of frequency charts depicted by Figures 2-5. Beside distribution of the output variable, these figures show the $90^{\text {th }}$ percentile, which is considered as high estimate of project duration.

The statistical distribution for the simulated base case is calculated with the given beta distribution and has a mean of 11.98 days and a median of 11.96 days. Since the base case scenario does not include risk events, competition time can be shorter than expected. As can be seen from Fig 2. the maximum simulated duration was 13.96 and high estimate of project duration is 12.82 days $(90 \%$ certainty). A slightly different situation is with scenario 1 that includes one risk event assigned to activity A with probability of $30 \%$ and 1-day impact. As can be seen form Fig. 3 single risk event scenario results with a mean project duration of 12.27 days, median of 12.25 days, maximum value 14.74 of days, and high estimate of project duration 13.39 days.

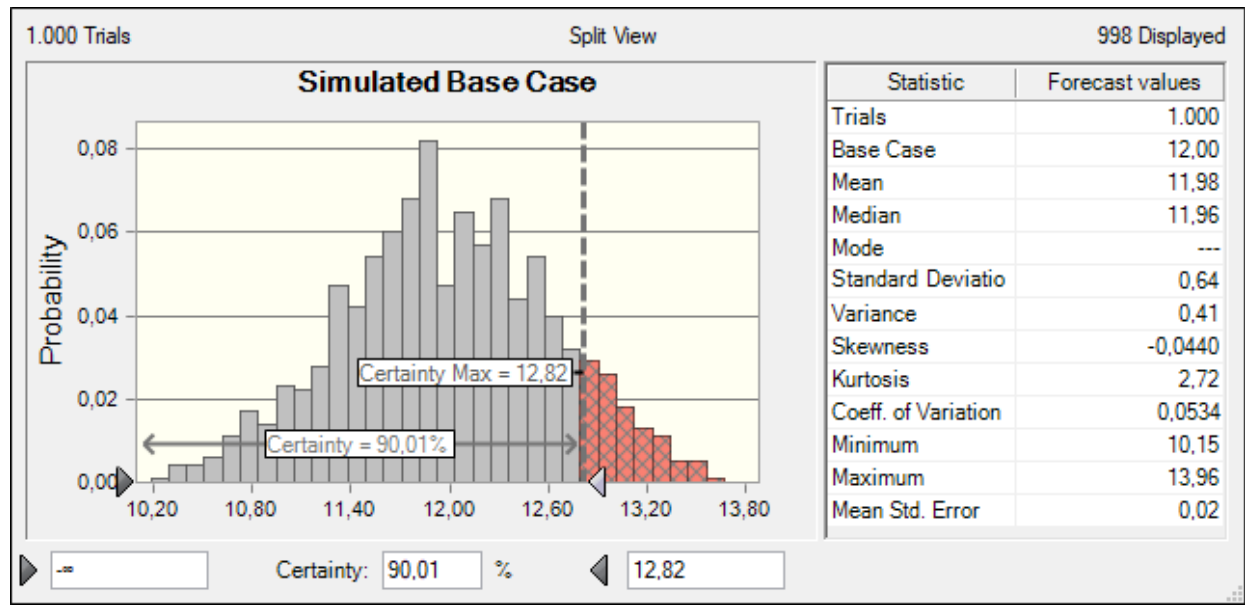

Figure 2: Simulated Base Case - high estimate of duration $\left(90^{\text {th }}\right.$ percentile) 


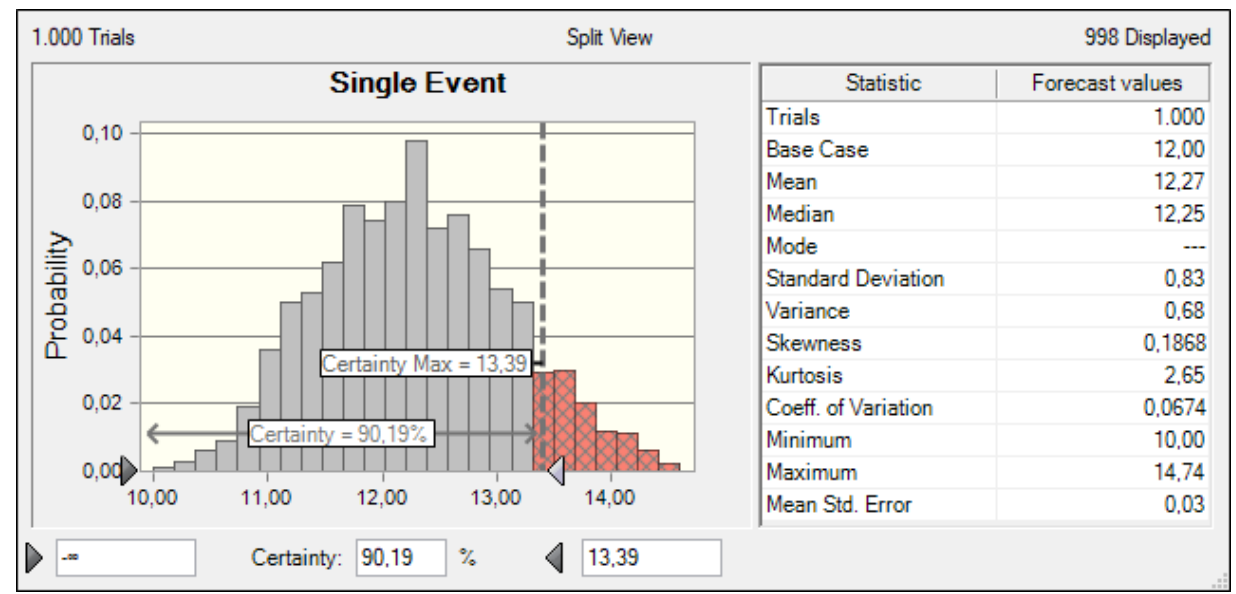

Figure 3: Single risk event - high estimate of duration $\left(90^{\text {th }}\right.$ percentile)

Due to the increased number of risk events, significantly different results were obtained in the last two scenarios. Fig. 4 shows a third scenario with three independent risk events, one related to each activity, each with probability of $30 \%$ and 1,2 , and 3 days of impact respectively. As can be seen form Fig. 4 , this multiple independent risk event scenario results with a mean project duration of 13.75 days, median of 13.53 days, and maximum value of 19.12 days.

The last scenario includes event chain with three interrelated risk events. Risk event related to activity A has a probability of $30 \%$, and potential impact of 1 day. If the first event occurs, other two represent certain events that extend the project duration of activity B for 2 days and activity $\mathrm{C}$ for 3 days. As can be seen form Fig. 5, event chain scenario results with a mean project duration of 15.24 days, median of 15.99 days, and maximum value of 19.89 days.

Bimodal distribution of the output variable makes the results of the last scenario significantly different from the result obtained in the multiple independent risk events scenario. High estimate of project duration in the event chain scenario is 18.35 days, which is more than 2 days longer than the high estimate of the project duration in the independent risk event scenario (16.33). These results unambiguously indicate that event chain leads to longer project competition time compared to the series of independent events with equal probability and impact.

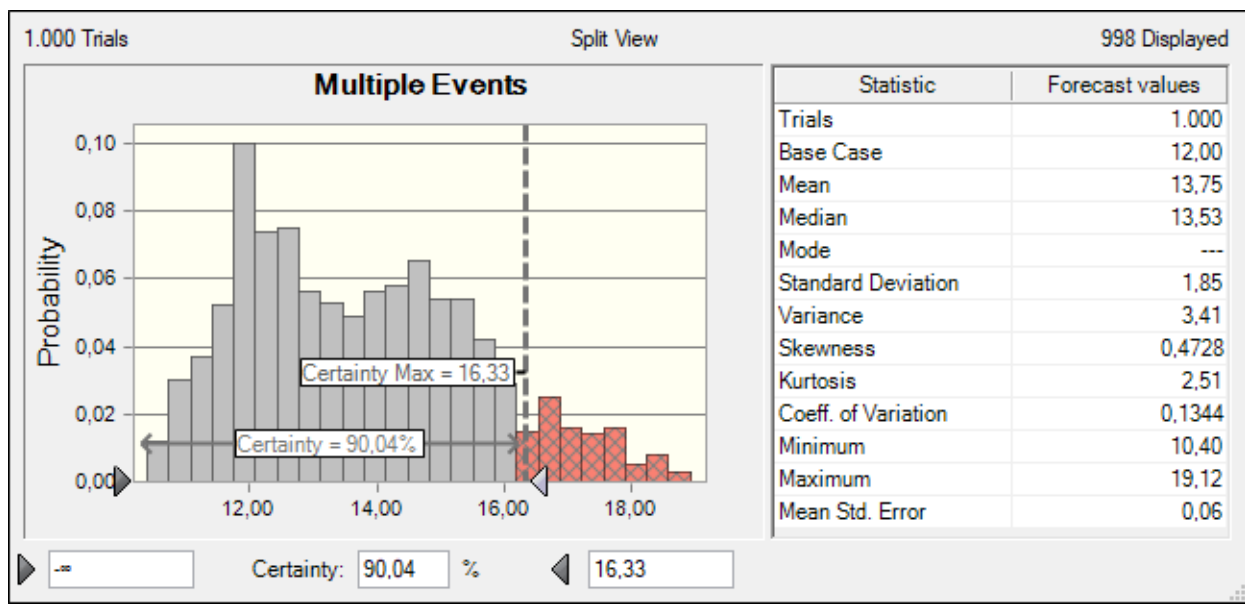

Figure 4: Multiple (independent) risk events - high estimate of duration $\left(90^{\text {th }}\right.$ percentile) 


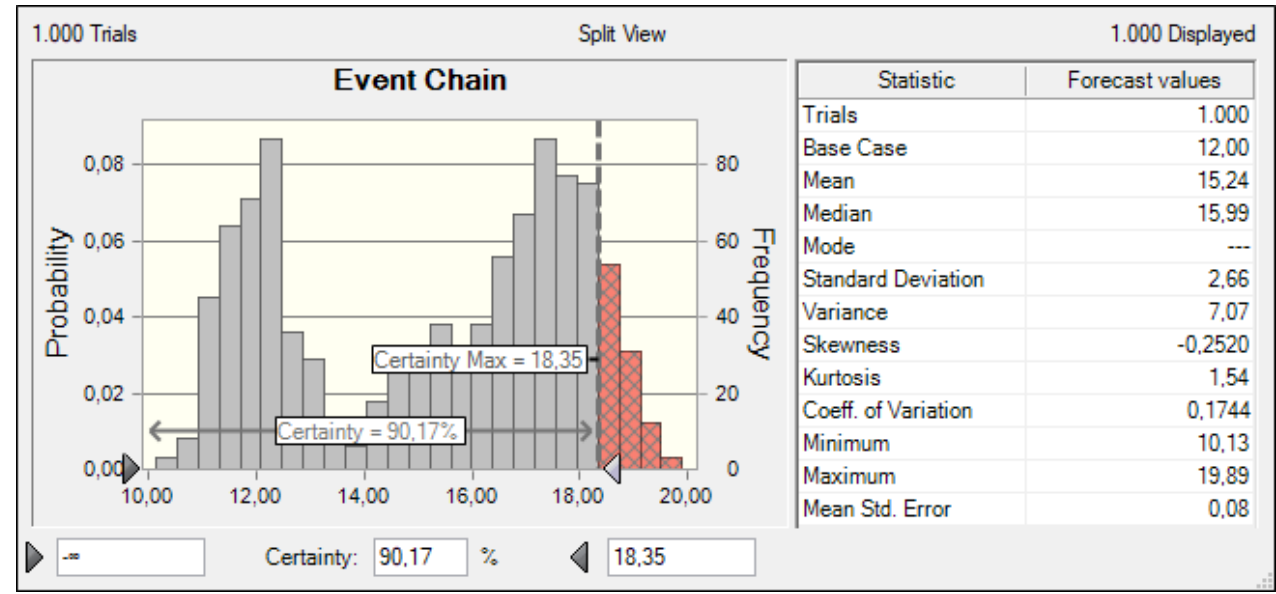

Figure 5: Event Chain statistics - high estimate of duration $\left(90^{\text {th }}\right.$ percentile $)$

Finally, sensitivity analysis was performed in order to identify the most critical events. This was done by computing the rank correlation coefficients between every assumption and every forecast. Figure 6 shows the sensitivity charts that rank the variables from the most important down to the least important in the examined scenarios. High correlation coefficients indicate that the variable significantly impacts project duration, and positive coefficients indicate that an increase in the variable leads to an increase of project duration.

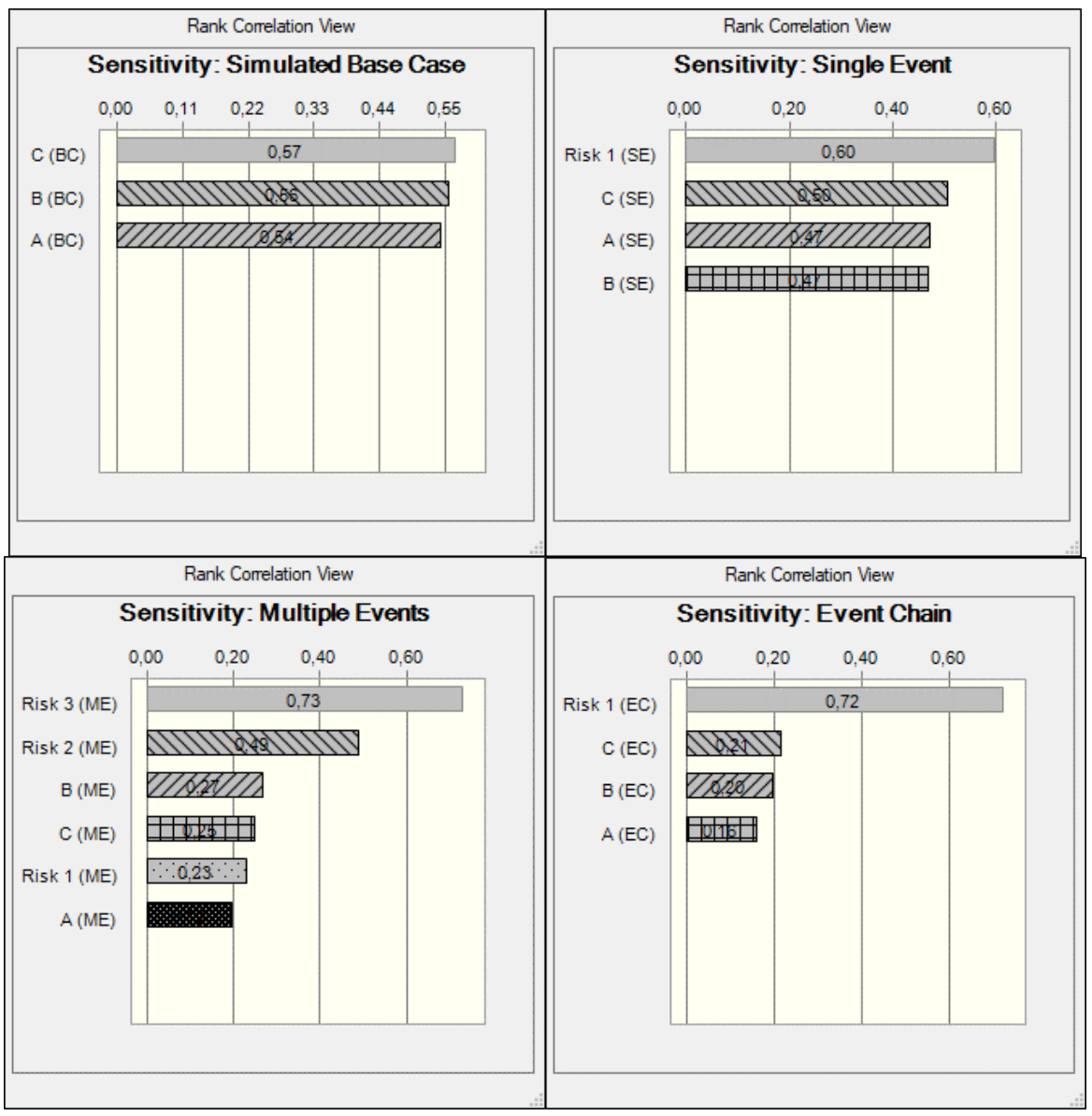

Figure 6: Sensitivity analysis for the examined project scenarios 
Since the activities are the only inputs in the first scenario and they use the same probability distribution (BetaPERT), sensitivity analysis output in the upper left corner seems logical. A similar situation is with the second scenario (upper right corner), where the single risk event is depicted as the most significant. In the scenario with three independent risk events with equal probabilities (lower left corner), the last risk event is the most significant as it involves the greatest potential delay. In this case, first risk event appears less significant than the regular variation in the duration of activities $\mathrm{B}$ and $\mathrm{C}$. Unlike the previous, the most important variable in the last scenario is the first risk event, which triggers the second and third risk event and at the same time significantly affects the project duration.

\section{CONCLUSION}

Existing literature has identified cognitive and motivational biases as the key reasons for poor project scheduling. Unlike the most traditional quantitative risk management methods, ECM suggest the use of additional factors such as interrelation between risk events, moments of risk occurrence, activity delays and repetitions, execution of risk response plan, etc. In this way, it seeks to reduce the impact of selective perception, overconfidence, availability heuristic and anchoring, as the most significant root causes of poor project time planning.

Starting from the original schedule baseline, ECM method upgrades the existing methods with additional information on risk events and their impacts and incorporates valuable historical information. As the project progresses and new information is discovered, the plan can be updated, increasing the accuracy of the estimates and the ability to control the project schedule. The complex relationships between project activities can be visualized using diagrams, which makes it easier to identify risk events, event chains and calculate their potential impact. ECM application is likely to increase with the ongoing expansion of international large-scale cross-sector projects, as it will provide a common risk assessment tool understandable to all parties involved throughout the project lifecycle.
The illustrative example was used to demonstrate the importance of identification and analysis of risk events and the simplicity of the ECM method, regardless of the type, size and complexity of the project. The application of the method does not require development and use of a specialized simulation software, so it is also possible to use existing tools for quantitative risk analysis. Therefore, some of the key benefits of the ECM described in this paper can be significant factor for greater application of this relatively novel approach and further integration with the existing project management practice.

\section{REFERENCES}

Agarwal, R., \& Virine, L. (2017). Monte Carlo Project Risk Analysis. In Raydugin, Y. (ed) Handbook of Research on Leveraging Risk and Uncertainties for Effective Project Management. IGI Global; 1 edition.

Avlijaš, G. (2019). Examining the value of Monte Carlo simulation for project time management. Management: Journal of Sustainable Business and Management Solutions in Emerging Economies, 24(1), 11-23.

Buehler, R., Griffin, D., \& Ross, M. (1994). Exploring the "planning fallacy": Why people underestimate their task completion times. Journal of Personality and Social Psychology, 67, 366-381.

Davis, R. (2008). Teaching note-Teaching project simulation in Excel using PERTbeta distributions. INFORMS Transactions on Education, 8(3), 139-148.

Evans, J., Barston, J. L., \& Pollard, P. (1983). On the conflict between logic and belief in syllogistic reasoning. Memory and Cognition, 11, 295-306.

Hulett, D. (2016). Practical schedule risk analysis. Routledge.

Intaver Institute Inc. (2011). Event Chain Methodology in Project Management [White paper]. Retrieved from: http://www.intaver.com/Articles/Article_ EventChainMethodology2011.pdf

Kardes, I., Ozturk, A., Cavusgil, S. T., \& Cavusgil, E. (2013). Managing global megaprojects: Complexity and risk 
management. International Business Review, 22(6), 905-917.

Lovallo, D., \& Kahneman, D. (2003). Delusions of success: how optimism undermines executives' decisions, Harvard Business Review, July Issue, pp. 56-63.

McCray, G. E., Purvis, R. L., \& McCray, C. G. (2002). Project Management Under Uncertainties: The Impact of Heuristics and Biases. Project Management Journal. Vol. 33, No. 1. 49-57.

Merrow, E. W. (2011). Industrial megaprojects. Hoboken, NJ: Wiley.

Plous, S. (1993). The Psychology of Judgment and Decision Making, McGraw-Hill.

PMI. (2017). A guide to the project management body of knowledge (PMBOK guide) (Vol. 6). Project Management Institute.

Scheinin, W., \& Hefner, R. (2005). A Comprehensive Survey of Risk Sources and Categories. In Proceedings of Space Systems Engineering and Risk Management Symposiums (pp. 337-350).

Schuyler, J. R. (2001). Risk and decision analysis in projects. Project Management Institute, Newton Square.
Virine, L., \& Rapley, L. (2003). Visualization of Probabilistic Business Models, In Proceedings of 2003 Winter Simulation Conference, New Orleans, LA.4

Virine, L., \& McVean, J. (2004). Visual Modeling of Business Problems: Workflow and Patterns, In Proceedings of 2004 Winter Simulation Conference, Washington DC.

Virine, L. , \& Trumper, M. (2015). Predicting the unpredictable: How to analyze project risks using event chain methodology. PM Network, 29(9), 28-29.

Virine, L. (2013). Integrated Qualitative and Quantitative Risk Analysis of Project Portfolios. In Proceedings of 2013 Enterprise Risk Management Symposium, April 22-24, Chicago, IL.

Virine, L., \& Trumper, M. (2019). Project decisions: the art and science. BerrettKoehler Publishers.

Williams, T. (2004). Why Monte Carlo simulations of project networks can mislead. Project Management Journal, 35(3), 53-61.

Wysocki, R. K., \& McGary, R. (2003). Effective Project Management: Traditional, Adaptive, Extreme, 3rd Edition, John Wiley \& Sons Canada, Ltd. 